\title{
Multimodality Imaging Approach in Evaluation of Post-Traumatic Bronchobiliary Fistulas
}

\author{
Savas Ozdemir ${ }^{1}$, Ashley Way ${ }^{1}$, Dheeraj Gopireddy ${ }^{1}$ \\ 1. Radiology, University of Florida College of Medicine, Jacksonville, USA
}

Corresponding author: Savas Ozdemir, savas.ozdemir@jax.ufl.edu

\begin{abstract}
Post-traumatic bronchobiliary fistulas (BBF) are extremely rare with high morbidity and mortality rates. Accurate and timely diagnosis of these entities is critical for appropriate treatment, which usually requires a multidisciplinary approach. We describe two post-traumatic cases using a multimodality approach including computed tomography (CT), magnetic resonance imaging (MRI)/Magnetic Resonance

Cholangiopancreatography (MRCP), and hepatobiliary scintigraphy with specific emphasis on the imaging features for each modality. Management of hepatobiliary fistulas is complex, involving extensive diagnostic work up followed by a conservative and/or surgical approach.
\end{abstract}

Categories: Radiology

Keywords: post-traumatic bronchobiliary fistula, hepatobiliary scintigraphy, spect/ct, computed tomography, magnetic resonance imaging

\section{Introduction}

Bronchobiliary fistula (BBF) is communication of biliary system and bronchial tree. It was first described by Peacock in 1850 [1]. Common causes of acquired BBF are hepatic neoplasms, bile duct obstruction, cholangiolithiasis, hepatic hydatidosis, trauma and chronic pancreatitis [2]. Post-traumatic BBFs are extremely rare. Computed tomography (CT) is used for initial evaluation of liver and biliary system in patients with history of penetrating trauma, providing critical anatomical information on the pattern of hepatic and biliary injuries. Although magnetic resonance imaging (MRI)/magnetic resonance cholangiopancreatography (MRCP) is not the first line of imaging in the acute trauma setting, it is utilized acutely when initial CT demonstrate signs of bile leak [3]. Hepatobiliary scintigraphy is very useful in evaluation of suspected BBFs [4-6]. One of the advantages of hepatobiliary scintigraphy is ability to obtain sequential dynamic functional images for a prolonged time. Bile movement can be visualized in real time to confirm the communication between biliary system and bronchial tree. Additional imaging with single positron emission computed tomography (SPECT/CT) may reveal exact location of fistulous communications, therefore confirming the diagnosis with high diagnostic accuracy [5].

Received 08/19/2020 Review began 08/24/2020 Review ended 08/29/2020 Published 08/31/2020

\section{(c) Copyright 2020}

Ozdemir et al. This is an open access article distributed under the terms of the Creative Commons Attribution License CC-BY 4.0., which permits unrestricted use, distribution, and reproduction in any medium, provided the original author and source are credited.

\section{Case Presentation}

\section{Case 1}

A 16-year-old male with history of penetrating injury (gunshot) to the right upper quadrant and right lower hemithorax complicated by biliary leak, diaphragmatic injury, and bilomas. Initial contrast-enhanced CT demonstrated multiloculated fluid collections in the right subphrenic space and small pleural effusion (Figure $1 A, 1 B$ ). This finding was also further confirmed with abdominal MRI and MRCP, which demonstrated a direct communication between the anterior division of the right hepatic duct and right pleural space (Figure $1 C, 1 D$ ). These collections were drained percutaneously and fluid analysis demonstrated infected bilomas. However, the patient developed bilioptysis which was confirmed with bronchoscopy and fluid analysis. Subsequently, a hepatobiliary scintigraphy was positive for BBF which confirmed abnormal communication at the level of the distal right lower lobe bronchus (Figure $1 E$ ). The patient was managed with multiple biliary drains and stents to aid in decompression of the biliary system with subsequent resolution of the bronchobiliary fistula.

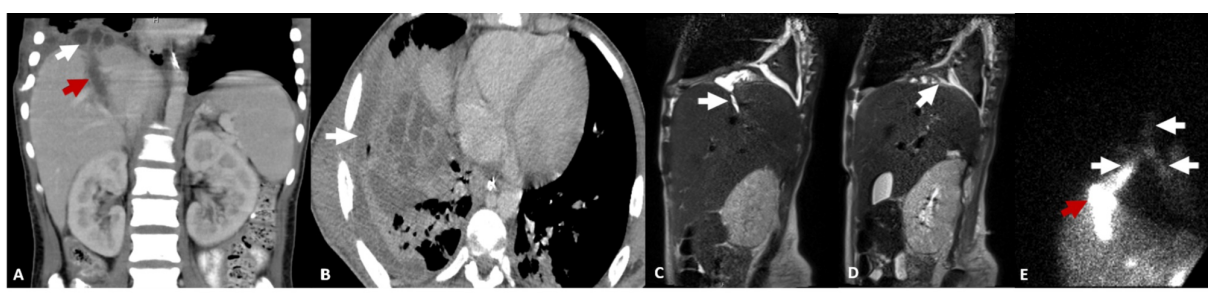

FIGURE 1: A 16-year-old male with history of penetrating injury 
(gunshot) to the right upper quadrant and right lower hemithorax complicated by biliary leak, diaphragmatic injury, and bilomas.

(A) Coronal contrast-enhanced CT shows a fluid filled track (red arrow) and subdiaphragmatic multiloculated fluid collection (white arrow). (B) Axial contrast-enhanced CT shows a subdiaphragmatic multiloculated fluid collection (white arrow). (C) Sagittal Single Shot Fast Spin ECHO (SSFSE) images show a biliary tract extending to subdiapragmatic fluid collection (white arrow) and (D) communication between the subhepatic fluid collection and pleural cavity (white arrow). (E) Anterior planar image of hepatobiliary scintigraphy shows a focal area of increased activity in the subdiaphragmatic region, consistent with biloma (red) and abnormal activity in the bronchial tree, consistent with bronchobiliary fistula (white arrows).

\section{Case 2}

A 40-year-old male and victim of gun violence with surgical history of laparotomy, left hepatectomy, cholecystectomy and removal of two foreign bodies. Six days later, he underwent an endoscopic retrograde cholangiopancreatography (ERCP) which revealed extravasation of contrast from the common bile duct and he returned to the operating room for drainage of intraabdominal abscesses and a Roux-en-Y hepaticojejunostomy.

Since then over several months he suffered from many hepatic abscesses, bilomas, cholangitis, and gram negative bacteremia, primarily managed with percutaneous drains and antibiotics. His clinical course was further complicated by misplaced drains and a biliarycutaneous fistula.

Multiple admissions were made for management of the biliarycutaneous fistula, a most recent CT of the abdomen and pelvis demonstrated loculated collection in the hepatic dome extending into the right subdiaphragmatic space (Figure 2A, 2B). This was also confirmed on MRI of the abdomen (Figure 2C, 2D). His course was further managed with a percutaneous CT guided drainage which revealed an infected biloma. A hepatobiliary scintigraphy (Figure 3A) and SPECT/CT (Figure 3B, 3C, 3D, 3E) confirmed progressive accumulation of activity extending into the right subhepatic space, right hemithorax/bronchial system and anterior abdominal wall in the epigastric region, consistent with bile leak to the subhepatic region, bronchobiliary fistula and biliarycutaneous fistula. Patient was found to have pseudomonas in culture. He was discharged to complete extended course of antibiotic treatment by home infusion.

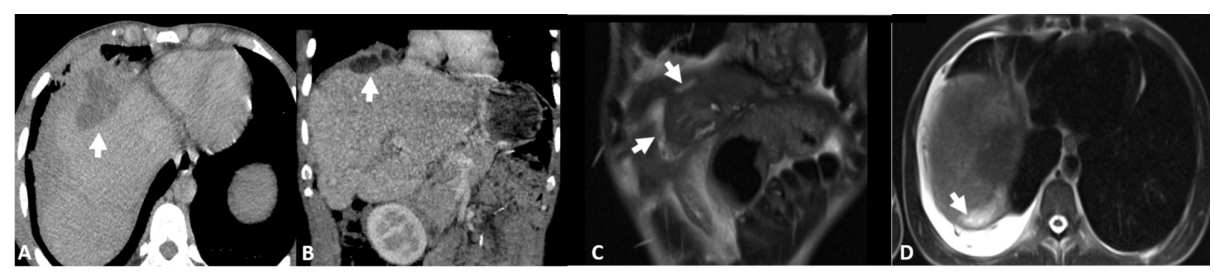

FIGURE 2: A 40-year-old male and victim of gun violence with surgical history of laparotomy, left hepatectomy, cholecystectomy and removal of two foreign bodies.

(A) Axial and (B) coronal contrast-enhanced CT show a subdiaphragmatic multiloculated fluid collection (white arrows). (C) Coronal Single Shot Fast Spin ECHO (SSFSE) image shows biliary tract extending to subdiaphragmatic fluid collection (white arrows). (D) Axial SSFSE image shows communication between the subhepatic fluid collection and pleural cavity (white arrow).

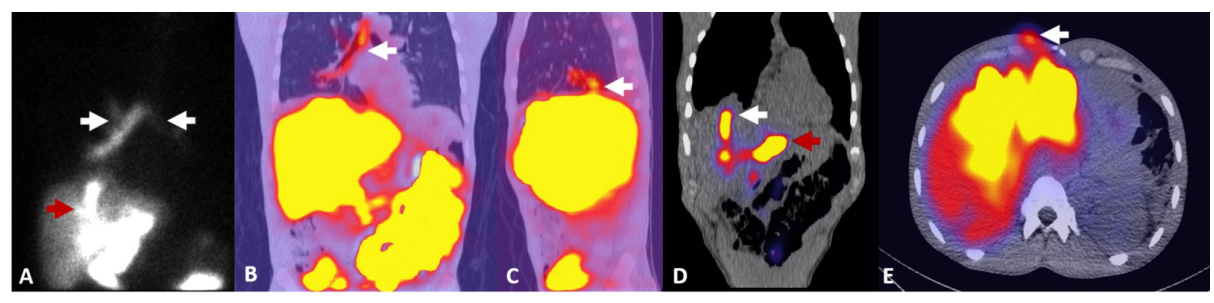

FIGURE 3: A 40-year-old male and victim of gun violence with surgical history of laparotomy, left hepatectomy, cholecystectomy and removal of two foreign bodies.

(A) Anterior planar image of hepatobiliary scintigraphy shows abnormal activity which partially fills subdiaphragmatic fluid collection (red arrow) and abnormal activity in the bronchial tree, consistent with 
bronchobiliary fistula (white arrows). (B) Coronal SPECT/CT shows activity in the bronchial tree (white arrow). (C) Sagittal SPECT/CT reveals location of bronchobiliary fistula (white arrow). (D) Coronal SPECT/CT shows subhepatic biliary fluid collection (red arrow) and extention of biliary tract to hepatic dome (white arrow). (E) Axial SPECT/CT reveals biliarycutaneus fistula (white arrow).

\section{Discussion}

There is paucity of large case series in literature since post-traumatic BBFs are extremely uncommon. Most of the publications consists of case reports and small series. A systematic literature review of 68 cases published in 30 years by Liao et al. revealed only seven cases (10.2\%) secondary to trauma [2].

A significant clinical symptoms in patients with BBFs is bilioptysis. Bilioptysis is presence of bile in the sputum and believed to be pathognomonic finding for BBFs. Other common symptoms include irritating cough, fever, and jaundice [2].

Penetrating injuries rather than blunt traumas are reported in etiology of bronchopulmonary fistulas [7]. Both of our cases are result of penetrating gunshot wounds to the right upper quadrant.

Initial evaluation usually starts with CT which is useful to demonstrate liver injury, abdominal and thoracic fluid collections, as well as abnormal lung findings. The widespread availability, decreased variability between operators, imaging speed, and relatively few contraindications make it ideal in immediate traumatic assessment [3,8]. However, it rarely depicts BBF directly [4]. Instead, CT reveals several indirect findings characteristic of a biliary injury which may include perihepatic or intrahepatic fluid collections and ascites in acute phase, progressive growth of perihepatic or intraparenchymal fluid collection on follow-up imaging, and/or other secondary signs of biliary injury [3]. Diaphragmatic disruption caused by laceration associated with right pleural effusion must raise the possibility of biliarypleural fistula [9]. Additionally, CT is also helpful in management of these complex post-traumatic collections for placement of external drains.

Conventional MRI of the abdomen with MRCP (Magnetic Resonance Cholangiopancreatography) can provide good anatomical information of the biliary system. However, contrast-enhanced magnetic resonance cholangiography using hepatobiliary contrast agents was reported to demonstrate bronchobiliary fistula since it depicts biliary excretion from injured ducts [10]. Unfortunately, limitations of MRI still exist including weak/variable filing of bile ducts secondary to reduced hepatic function, potential interference with T2-weighted images secondary to T2 shortening effect, as well as general contraindications to MRI including certain implanted cardiac devises or bullet fragments in the setting of trauma [11].

Hepatobiliary scintigraphy, similar to MR cholangiopancreatography with hepatobiliary contrast agents provide functional information. Image acquisition may be obtained for a prolonged time to trace bile movement. The precise location of bronchobiliary connection could not be confidently determined with this method even when SPECT was performed in the past. SPECT/CT as a hybrid imaging technique combining the functional information of hepatobiliary scintigraphy and anatomical information of CT overcomes the shortcoming and may clearly define fistulous tract [5].

Management of BBF varies from a conservative approach to definitive surgical management. The conservative approach is preferred in the initial management of bile leaks. Biliary decompression or diversion using endoscopic sphincterotomy, biliary stent placement, or nasobiliary drainage and percutaneous image-guided catheter collections alone may be successful. More definitive treatment would require surgical resection of the fistula with pulmonary segmentectomy, especially in the presence of lung injury [12-15]. Recently, more advanced and less invasive approaches are being tried including transhepatic embolization of the fistulas [16].

\section{Conclusions}

A multimodality approach is critical for stepwise diagnosis of BBFs. Although CT is used in initial evaluation of BBFs, hepatobiliary scintigraphy can confirm biliary leaks and differentiate bilomas from other fluid collections. Bile excretion and movement may be traced by sequential dynamic imaging. SPECT and more recently SPECT/CT may reveal connections between the bile ducts, bilomas and other structures such as bronchial tree and may play an important role in management of complex cases. MR cholangiopancreatography with hepatobiliary contrast agents also allows dynamic biliary imaging. It is an alternative to hepatobiliary scintigraphy and SPECT/CT and may demonstrate fistulous communications of the biliary system with high accuracy.

\section{Additional Information}

\section{Disclosures}

Human subjects: Consent was obtained by all participants in this study. Conflicts of interest: In compliance with the ICMJE uniform disclosure form, all authors declare the following: Payment/services 
info: All authors have declared that no financial support was received from any organization for the submitted work. Financial relationships: All authors have declared that they have no financial relationships at present or within the previous three years with any organizations that might have an interest in the submitted work. Other relationships: All authors have declared that there are no other relationships or activities that could appear to have influenced the submitted work.

\section{References}

1. Peacock TB: Case in which hydatids were expectorated, and one of suppuration in a hydatid cyst of the liver communicating with the lungs. Edinb Med Surg J. 1850, 74:33-46.

2. Liao GQ, Wang H, Zhu GY, Zhu KB, Lv FX, Tai S: Management of acquired bronchobiliary fistula: a systematic literature review of 68 cases published in 30 years. World J Gastroenterol. 2011, 17:3842-3849. 10.3748/wig.v17.i33.3842

3. Melamud K, LeBedis CA, Anderson SW, Soto JA: Biliary imaging: multimodality approach to imaging of biliary injuries and their complications. Radiographics. 2014, 34:613-623. 10.1148/rg.343130011

4. Andalkar L, Trow TK, Motroni B, Katz DS: Bronchobiliary fistula as a complication of liver metastases: diagnosis by HIDA scan. Clin Nucl Med. 2004, 29:289-291. 10.1097/01.rlu.0000122799.34030.9e

5. Bhattacharya A, Kamaleshwaran KK, Balasubramaniam Harisankar CN, Manohar K, Abrar ML, Mittal BR: Post-traumatic biliobronchial fistula demonstrated on hepatobiliary scintigraphy with SPECT/CT . Eur J Nucl Med Mol Imaging. 2010, 37:1994. 10.1007/s00259-010-1544-8

6. Parghane RV, Phulsunga RK, Gupta R, Basher RK, Bhattacharya A, Mittal BR: Usefulness of tc99mmebrofenin hepatobiliary scintigraphy and single photon emission computed tomography/computed tomography in the diagnosis of bronchobiliary fistula. World J Nucl Med. 2017, 16:317-319. 10.4103/14501147.21549

7. Andrade-Alegre R, Ruiz-Valdes M: Traumatic thoracobiliary (pleurobiliary and bronchobiliary) fistula. Asian Cardiovasc Thorac Ann. 2013, 21:43-47. 10.1177/0218492312454667

8. Patel NB, Oto A, Thomas S: Multidetector CT of emergent biliary pathologic conditions . Radiographics. 2013, 33:1867-1888. 10.1148/rg.337125038

9. Feld R, Wechsler RJ, Bonn J: Biliary-pleural fistulas without biliary obstruction: percutaneous catheter management. AJR Am J Roentgenol. 1997, 169:381-383. 10.2214/ajr.169.2.9242739

10. Karabulut N, Cakmak V, Kiter G: Confident diagnosis of bronchobiliary fistula using contrast-enhanced magnetic resonance cholangiography. Korean J Radiol. 2010, 11:493-496. 10.3348/kjr.2010.11.4.493

11. Yeh BM, Liu PS, Soto JA, Corvera CA, Hussain HK: MR imaging and CT of the biliary tract . Radiographics. 2009, 29:1669-1688. 10.1148/rg.296095514

12. Gandhi N, Kent T, Kaban JM, Stone M, Teperman S, Simon R: Bronchobiliary fistula after penetrating thoracoabdominal trauma: case report and literature review. J Trauma. 2009, 67:143-145. 10.1097/TA.0b013e318123fc34

13. Ball CG, Trexler S, Rajani RR, Vercruysse G, Feliciano DV, Nicholas JM: Importance of liver drainage in biliary-bronchopleural fistula resulting from thoracoabdominal gunshot injury. Can J Surg. 2009, 52:12-13.

14. Nantais J, Skelhorne-Gross G, Jimenez C, Ahmed N, Gomez D: Bilioptysis due to a single transcavitary thoracoabdominal gunshot wound. Trauma Surg Acute Care Open. 2020:000425-2020. 10.1136/tsaco-2019000425

15. Banerjee N, Rattan A, Priyadarshini P, Kumar S: Post-traumatic bronchobiliary fistula. BMJ Case Rep. 2019:228294-2019. 10.1136/bcr-2018-228294

16. Mehrzad H, Aziz A, Mangat K: Transhepatic embolisation of a traumatic broncho-biliary fistula: a novel approach. BMJ Case Rep. 2012, 2012:bcr2012006702. 10.1136/bcr-2012-006702 